\title{
An Integrated Genetic Algorithm and Integer Programming Approach to the Network Design Problem with Relays
}

\author{
Abdullah Konak ${ }^{1}$ and Sadan Kulturel-Konak ${ }^{2}$ \\ ${ }^{1}$ Penn State Berks, Information Sciences and Technology, \\ Tulpehocken Road P.O. Box 7009 Reading, PA 19610 \\ konak@psu.edu \\ ${ }^{2}$ Penn State Berks, Management Information Systems, \\ Tulpehocken Road P.O. Box 7009 Reading, PA 19610 \\ sadan@psu .edu
}

\section{Extended Abstract}

The network design problem with relays (NDPR) arises in many real-life telecommunication and supply chain networks. Similar to the multi-commodity network design problem, a set of commodities is given, and each commodity $k$ is to be routed through single path from the source node $s(k)$ to the sink node $t(k)$. However, an upper bound $\lambda$ is imposed on the distance that a commodity $k$ can travel on the path from the source node $s(k)$ to the sink node $t(k)$ without visiting special nodes, called relays. For example, in digital telecommunication networks, relays represent points where attenuated communication signals are regenerated. A fixed cost of $f_{i}$ is incurred when a node $i$ is dedicated as a relay, and each edge $(i, j)$ has an installation cost of $c_{i, j}$ and a length of $d_{i, j}$. The NDPR is defined as selecting a set of edges from a given set of candidate edges and determining relay nodes to minimize the network design cost while making sure that each commodity $k$ is routed through a single path on which the distances between the node $s(k)$ and the first relay, between any consecutive relays, and between the last relay and the node $t(k)$ are less than the upper bound $\lambda$.

This research first presents a new path-based formulation of the NDPR. Based on this formulation, it is shown that for a given set of commodity paths, the optimal location of relays can be determined by solving a set covering problem. Then, this approach is used in a genetic algorithm (GA) to solve the NDPR in two phases. Using a specialized crossover-mutation operator, the GA searches for a path for each commodity, and the relay locations are determined by solving the corresponding set covering problem. The proposed approach is extensively tested on several problems. Comparisons with the existing exact and heuristic approaches show that the proposed GA is very effective. Further research may focus on how to extend the proposed approach to solve the two-edge disjoint NDPR or the capacitated version of the problem. 